\title{
Mechanism-Based Design of Quinoline Potassium Acyltrifluoroborates (KATs) for Rapid Amide-Forming Ligations at Physiological pH
}

\author{
Matthias Tanriver ${ }^{\dagger}$, Yi-Chung Dzeng ${ }^{\dagger}$, Erwin Lam, Jeffrey W. Bode* \\ Laboratorium für Organische Chemie, Department of Chemistry and Applied \\ Biosciences, ETH Zürich, Zürich 8093, Switzerland
}

\begin{abstract}
Potassium acyltrifluoroborates (KATs) undergo chemoselective amide-forming ligations with hydroxylamines. Under aqueous, acidic conditions these ligations can proceed rapidly, with rate constants of $\sim 20 \mathrm{M}^{-1} \mathrm{~s}^{-1}$. The requirement for lower $\mathrm{pH}$ to obtain the fastest rates, however, limits their use with certain biomolecules and precludes in vivo applications. By mechanistic investigations into the KAT ligation, including kinetic studies, X-ray crystallography, and DFT calculations, we have identified a key role for a proton in accelerating the ligation. We applied this knowledge to the design and synthesis of 8-quinolyl acyltrifluoroborates, a new class of KATs that ligates with hydroxylamines at $\mathrm{pH} 7.4$ with rate constants $>4 \mathrm{M}^{-1} \mathrm{~s}^{-1}$. We trace the enhanced rate at physiological $\mathrm{pH}$ to unexpectedly high basicity of the 8-quinoline-KATs, which leads to their protonation even under neutral conditions. This proton assists the formation of the key tetrahedral intermediate and activates the leaving groups on the hydroxylamine towards a concerted 1,2- $\mathrm{BF}_{3}$ shift that leads to the amide product. We demonstrate that the fast ligations at $\mathrm{pH} 7.4$ can be carried out with a protein substrate at micromolar concentrations.
\end{abstract}

\section{Introduction}

Potassium acyltrifluoroborates (KATs) are robust, bench stable compounds that undergo amideforming ligations with $O$-substituted hydroxylamines under dilute, aqueous conditions. ${ }^{1-3}$ KAT ligation has already found applications in protein PEGylation, hydrogel formation and modification, and post-polymerization modification. ${ }^{4-7}$ The reaction is exceptionally fast at lower $\mathrm{pH}$, with rate constants $>20 \mathrm{M}^{-1} \mathrm{~s}^{-1}$ at $\mathrm{pH}$ 2, but becomes slower as the $\mathrm{pH}$ is increased. ${ }^{8}$ At $\mathrm{pH} 7$, amide 
formation can still proceed but at rates that are not suitable for reactions at micromolar concentrations. We have previously employed 2-pyridine-derived KATs as they were superior to aryl derivatives at $\mathrm{pH} 3-6,{ }^{4}$ with rate constants of $\sim 10 \mathrm{M}^{-1} \mathrm{~s}^{-1}$ at $\mathrm{pH}$ 3.6. These improved substrates, however, showed only modest reaction rates at physiological $\mathrm{pH}$, limiting their use for many desirable applications of chemoselective ligations. ${ }^{9,10}$

In this report we document the synthesis and rapid amide-forming ligations of 8-quinoline-derived KATs, which undergo KAT ligation at $\mathrm{pH} 7.4$ with second order rate constants up to $4 \mathrm{M}^{-1} \mathrm{~s}^{-1}$ more than 300 times faster than aryl derivatives. This report also provides detailed rate constants of various KAT derivatives at both $\mathrm{pH} 7.4$ and $\mathrm{pH} 3.8$. DFT calculations on the protonated and unprotonated reaction pathways provide insights into the mechanistic basis for the role of a proton in accelerating the KAT ligation. Characterization of the pyridyl- and quinolyl KATs - including titrations and X-ray crystallography - confirm the unexpectedly high basicity of the quinolyl KATs, which translates to superior reaction rates at higher $\mathrm{pH}$.

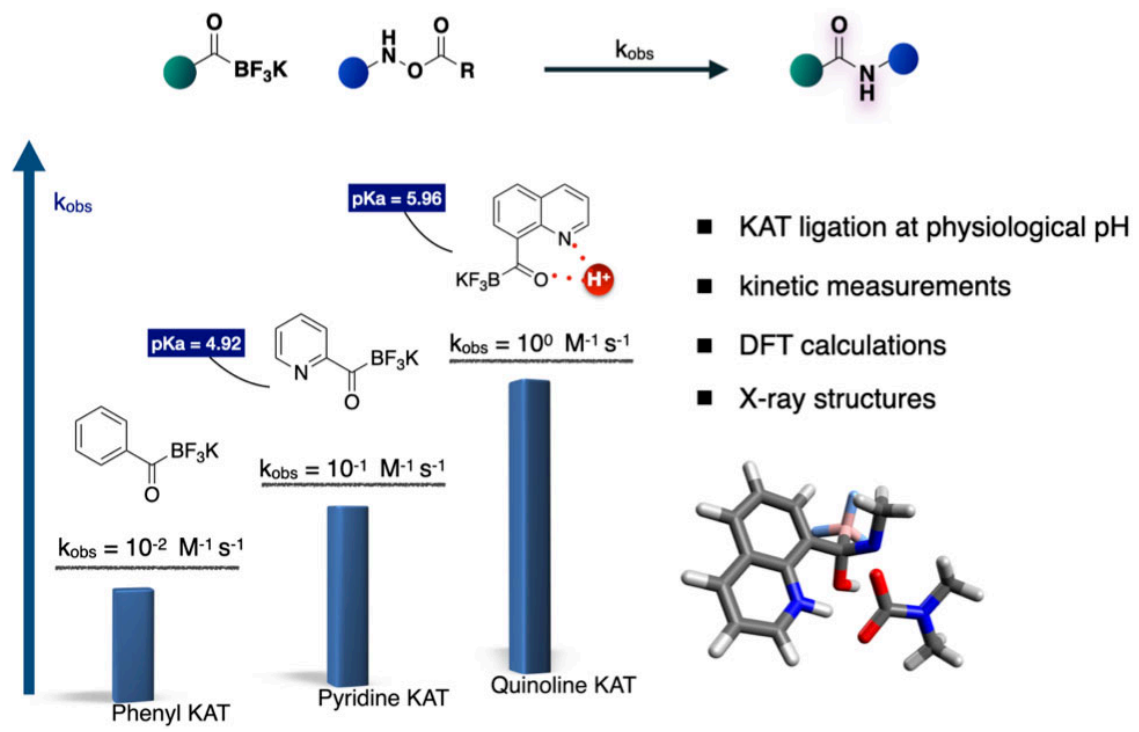

Scheme 1. Mechanism-based rate enhancement of KAT ligation.

\section{Results and Discussion}

Synthesis of KAT substrates. While initially challenging to produce, synthetic access to KATs has expanded markedly in the past few years.2, 11-19 These advances have allowed us to prepare a broad range of KATs containing a diverse range of functional groups and structural features. 
Based on our previous finding of superior ligation rates with pyridyl KATs, which we attributed to protonation of the pyridine nitrogen under acidic conditions, we aimed to synthesize a series of pyridyl- and quinolyl KATs that contain a basic atom near the reactive KAT group. Exploration of a number of hypotheses led to the initial finding that 8-quinolyl KATs performed better at higher $\mathrm{pH}$, and we elected to systematically study this class of compounds further - along with the aryl and pyridyl KATs - with regard to the role of electron-donating $(-\mathrm{OMe})$ and withdrawing $(-\mathrm{Cl})$ groups incorporated onto the aromatic rings. ${ }^{20,21}$ Quinolines are typically less basic than pyridines $\left(\mathrm{pK}_{\mathrm{a}}=4.9\right.$ for quinoline, $\mathrm{pK}_{\mathrm{a}}=5.2$ for pyridine $),{ }^{22}$ but place the basic nitrogen three, rather than two atoms away from the acylboronate carbonyl. ${ }^{23}$

Phenyl- and pyridyl KATs were synthesized from their corresponding halides using one equivalent of $n$-BuLi and reagent 1 each at $-78{ }^{\circ} \mathrm{C}$ in THF according to previous reports. ${ }^{11}$ Applying these conditions to 8-quinolyl halides, however, failed to yield any product and further optimizations were needed. Screening of reaction conditions showed that by conducting the lithium-halogen exchange at $-110{ }^{\circ} \mathrm{C}$ in a solvent mixture of diethyl ether and THF $(4: 1 \mathrm{v} / \mathrm{v})$ and allowing the reaction mixture to stir for one hour prior to the addition of reagent $\mathbf{1}$, followed by quenching at this temperature with aqueous KF and overnight mixing to form a precipitate provided access to the desired compounds (Scheme 2). 


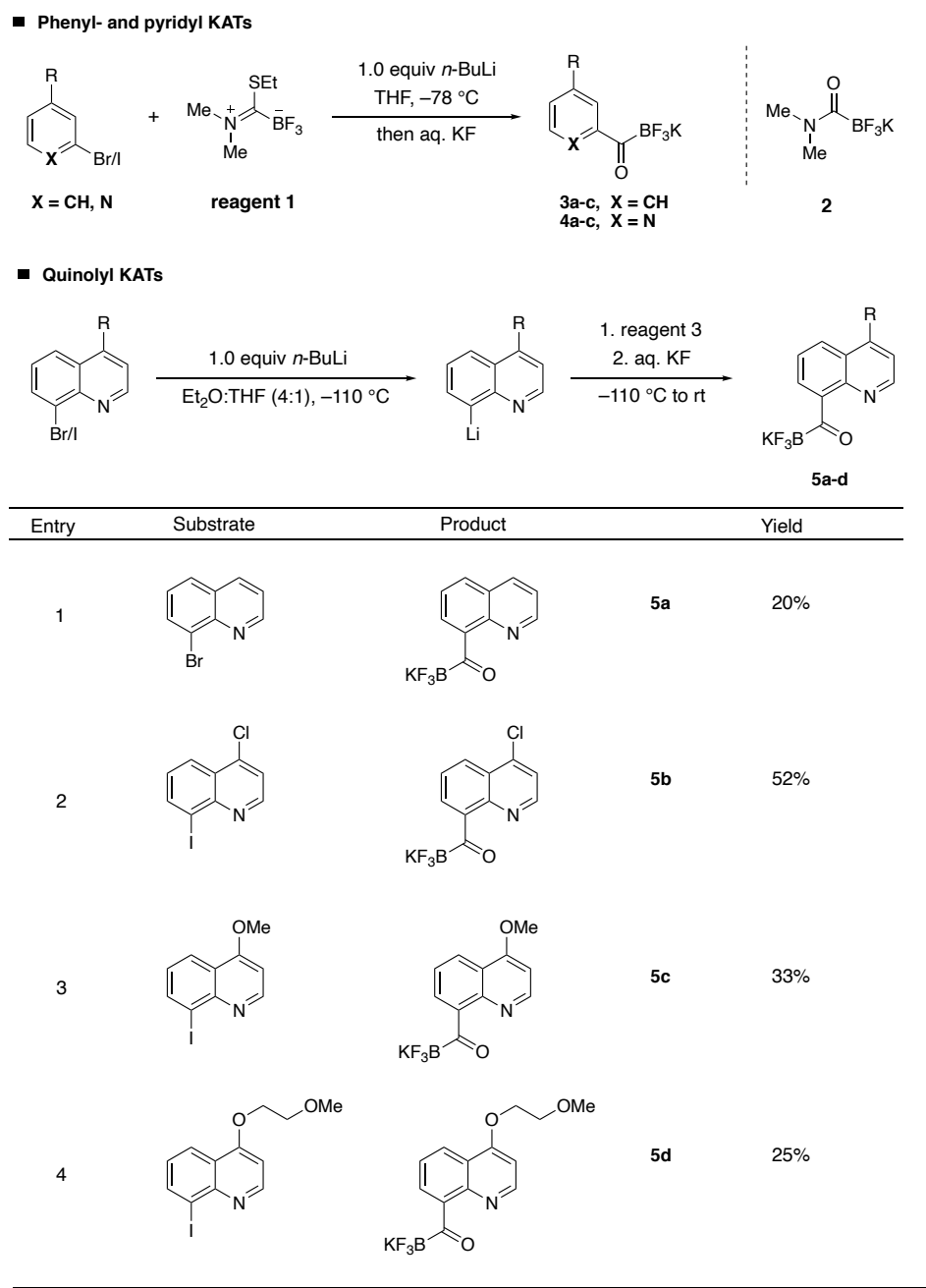

Scheme 2. All reactions were performed in an oven-dried Schlenk flask. $n$-BuLi was added dropwise over 30 min via syringe pump at $-78^{\circ} \mathrm{C}$ for phenyl- and pyridyl KATs and at $-110^{\circ} \mathrm{C}$ for quinolyl KATs; the reactions were quenched with 3.0 equiv $6.5 \mathrm{M} \mathrm{KF}_{(\mathrm{aq})}$ solution and the cooling bath was removed, allowing the reaction mixture to warm to rt.

Despite these improvements, the reaction between the 8-lithioquinolines and reagent 1 was sluggish and remaining reagent $\mathbf{1}$ formed a hydrolyzed side-product $\mathbf{2}$ that must be separated from the desired KAT. This could be accomplished by washing the crude mixtures with acetone to extract 2, as 8-quinolyl KATs, unlike most phenyl- and pyridyl KATs, do not dissolve in acetone. The desired KAT can be isolated from the remaining crude mixture using DMF, leaving behind the residual inorganic salts.

Kinetics of KAT ligations. With KAT substrates 3-5 in hand, we carefully evaluated the rates of their ligation with hydroxylamine 6 . For the determination of rate constants for KAT ligation, we 
selected UV-vis spectroscopy, as KATs have unique absorption bands that can be used for accurate determination of their concentration. We established calibration curves in buffered solutions at various $\mathrm{pH}$ values used for kinetic studies over the concentration range of $0-15 \mathrm{mM}$ (Figure 1b). Quinolyl KAT 5c failed to dissolve above $0.25 \mathrm{mM}$ in the buffers used for UV-vis measurements, therefore KAT $\mathbf{5 d}$ was synthesized and used instead.

(a)

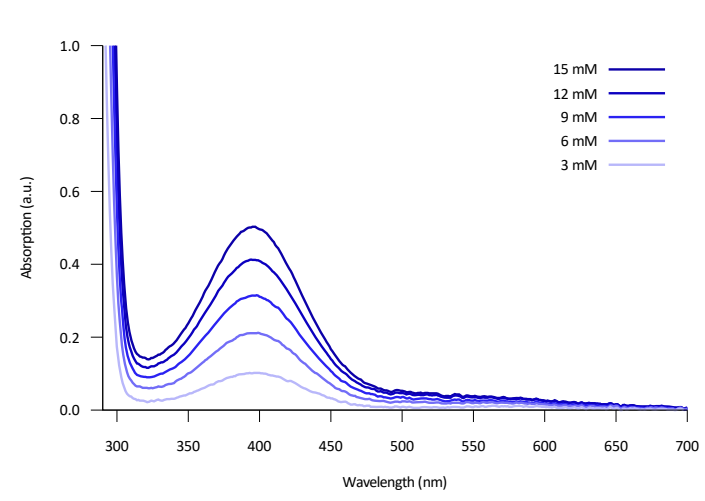

(c)

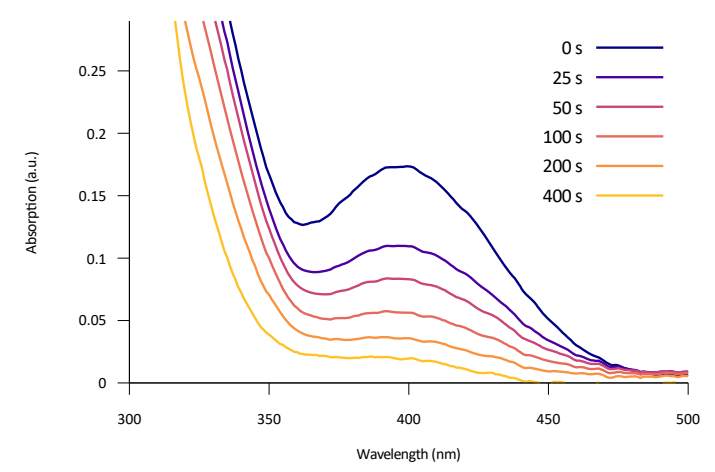

(b)

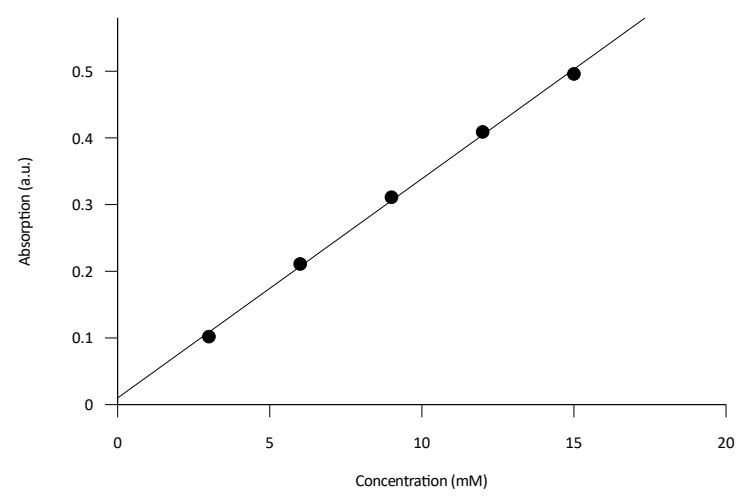

(d)

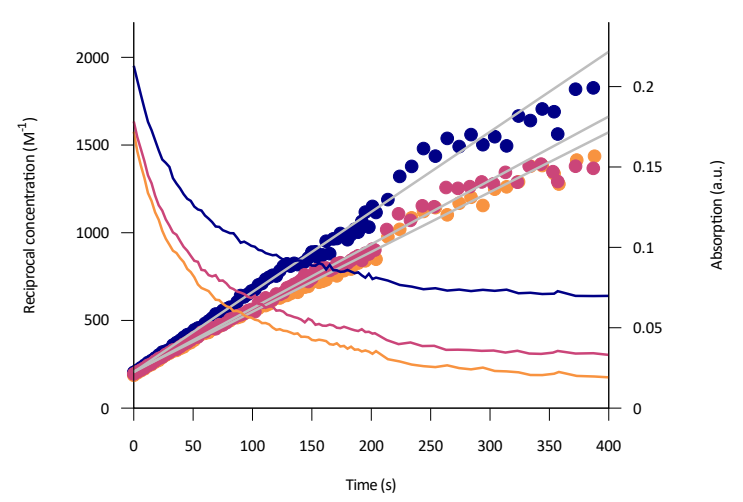

Figure 1. (a) Absorption spectra of KAT 4 b solutions at various concentrations at $\mathrm{pH}$ 3.8. (b) Absorption value of $\mathbf{4 b}$ at $400 \mathrm{~nm}$ plotted against concentration. The linear regression gave a slope of $32.87 \mathrm{M}^{-1} \mathrm{~cm}^{-1}$, intercept of 0.010 a.u. and $r^{2}$ of 0.9984 . All-wavelength regressions revealed the window of wavelengths that scales linearly with concentration. The absorption profile of the same KAT differs slightly under the two pH's used and required separate calibration as shown in the Supporting Information. (c) Decay of KAT specific absorption during the ligation reaction of $4 \mathrm{c}$ and 6 at $\mathrm{pH}$ 3.8. (d) Absorption time course data and regression plot used for determining the second order rate constant. Solid lines: absorption values. Circular dots: reciprocal concentration. Gray lines: linear fit to the reciprocal concentrations. 
We selected 1:1 (v/v) mixtures of acetonitrile with aqueous buffer solutions for the measurement of the ligation kinetics to ensure solubility of all reagents. KAT ligations work well in purely aqueous conditions, but these substrates required an organic co-solvent to ensure homogeneity throughout the course of the reaction at the various $\mathrm{pH}$ ranges and concentrations studied. To improve solubility of the starting materials and products, we designed and prepared water-soluble hydroxylamine 6. Potassium acetate buffer was employed for ligations at $\mathrm{pH} 3.8$, and potassium phosphate buffer was used for experiments at $\mathrm{pH}$ 7.4. The ligation reaction was initiated by mixing pre-buffered stock solutions of the KATs $(15 \mathrm{mM})$ with hydroxylamine 6 (15 mM) to give an initial concentration of $7.5 \mathrm{mM}$ in each reactant. UV-vis spectra were recorded over a period of $1000 \mathrm{~s}$ to monitor the consumption of KATs.

A representative set of UV absorption profiles over time is illustrated in Fig. 1c and shows the decay of the KAT specific band as the ligation progress. Approximately 100 time points were recorded over the 1000 seconds of measurement. The concentration of the KATs was determined by Beer's law using the determined molar absorption coefficients. Initial studies determined that the reaction is single order with respect to each reactant and the reaction was treated as an overall second order reaction with equimolar amounts of KAT and hydroxylamine. Linear regression of the reciprocal concentration values against time was used to determine the apparent second order rate constants (Figure 1d). With the uncertainty in absorbance measurement being as low as 0.001 a.u. in terms of standard deviation from blank measurements, the number of time points enabled the reliable determination of the background absorption as well as a reasonable fit to the assumed second order kinetics. Only the later portions of the data deviated slightly from ideal second order behavior. All measurements were repeated three times (marked in color) to calculate the averages and standard deviations of the rate constants. 
(a)

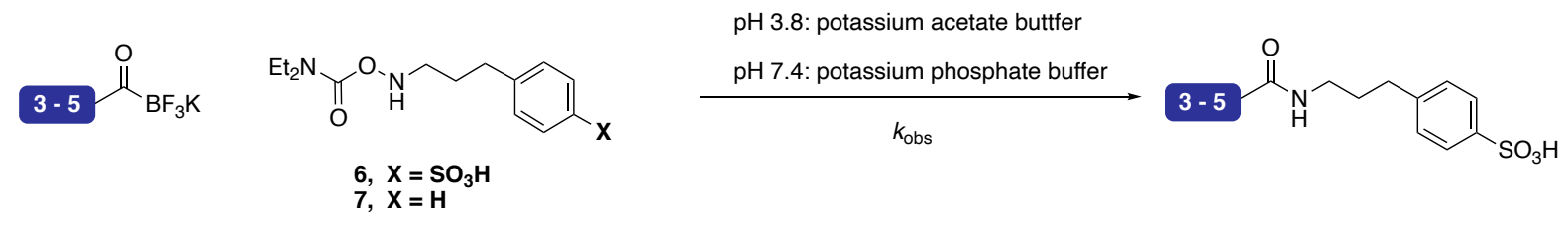

(b)

\begin{tabular}{cccc}
\hline \multirow{2}{*}{ Entry } & \multicolumn{2}{c}{$\boldsymbol{k}_{\text {obs }\left[\mathrm{M}^{-1} \mathbf{s}^{-1}\right]}$} & \multirow{2}{*}{$\boldsymbol{k}_{\text {rel, } \mathrm{pH} 7.4}$} \\
\cline { 2 - 3 } & $\mathrm{pH} 3.8$ & $\mathrm{pH} \mathrm{7.4}$ & \\
\hline 3a & 1.798 & 0.009 & 1.00 \\
3b & 3.328 & 0.015 & 1.67 \\
3c & 3.129 & 0.006 & 0.67 \\
& & & \\
4a & 13.82 & 0.030 & 3.34 \\
4b & 15.01 & 0.075 & 8.33 \\
4c & 3.874 & 0.150 & 16.7 \\
5a & 8.535 & 1.888 & 209 \\
5b & 9.579 & 2.440 & 271 \\
5d & 5.289 & 4.536 & 504 \\
\hline
\end{tabular}

Figure 2. (a) KATs and hydroxylamine 6 were mixed at equimolar concentrations $(7.5 \mathrm{mM})$ at $\mathrm{pH} 3.8$ and $\mathrm{pH} 7.4$. UV-vis measurements were carried out using a quartz

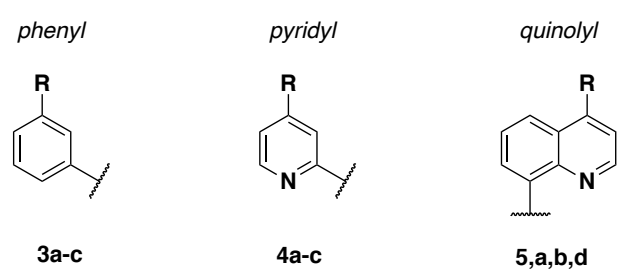

3a, $\mathrm{R}=\mathrm{H} \quad 4 \mathrm{a}, \mathrm{R}=\mathrm{H} \quad 5 \mathrm{a}, \mathrm{R}=\mathrm{H}$

$3 \mathbf{b}, \mathrm{R}=\mathrm{Cl} \quad \mathbf{4 b}, \mathrm{R}=\mathrm{Cl} \quad \mathbf{5 b}, \mathrm{R}=\mathrm{Cl}$

3c, $\mathrm{R}=\mathrm{OMe} \quad$ 4c, $\mathrm{R}=\mathrm{OMe} \quad \mathbf{5 d}, \mathrm{R}=\mathrm{O}\left(\mathrm{CH}_{2}\right)_{2} \mathrm{OMe}$ cuvette with a path length of $1 \mathrm{~cm}$. (b) Table and chart including error bars of calculated second order rate constants.

Figure $2 b$ summarizes the rate constants measured. KAT $3 a$, which has no rate-accelerating features, was taken as the reference of relative reactivity. At $\mathrm{pH} 7.4$, the 2-methoxyethoxysubstituted quinolyl KAT $\mathbf{5 d}$ had the highest rate constant, with a 500-fold acceleration over the reference. The dominant factor was the difference in aryl groups. Unsubstituted pyridyl KAT 4a reacted 3.3-fold faster, and quinolyl KAT 5a over 200 -fold faster than reference $3 \mathbf{a}$ at $\mathrm{pH} 7.4$. We were pleased to see that the presence of a basic nitrogen near the KAT group already led to a major change in reactivity. The other prominent factor was the substitution effect at the position opposite to the nitrogen atom. Within the pyridyl KAT 4 series at $\mathrm{pH} 7.4$, we observed a 16.7-fold 
increase in rate with a methoxy substituent, and an 8.3-fold increase with a chloro substituent. The same trend also held true within the quinolyl KAT 5 series. Electron-donating groups enhance the basicity of the nitrogen, which should lead to a higher proportion of the protonated forms. ${ }^{20}$ This would account for the fact that methoxy-substituted pyridyl KAT 4c and quinolyl KAT 5d were the most reactive ones in their respective aryl subgroups at $\mathrm{pH} 7.4$, but the slowest at $\mathrm{pH} 3.8$.

At $\mathrm{pH}$ 3.8, all of the KATs examined participated in the KAT ligation with second order rate constants higher than $1 \mathrm{M}^{-1} \mathrm{~s}^{-1}$, but the alkoxy-substituted pyridine $\mathbf{4 c}$ and quinoline $\mathbf{5 d}$ were slower than their analogs. This was consistent with the expectation that all pyridyl- and quinolyl KATs studied would be protonated at $\mathrm{pH} 3.8$ and inductive substituent effects that modulate the electrophilicity of the KAT dominated. Overall, the most reactive KAT at pH 3.8 was chloropyridyl KAT 4b. This confirms that, as expected, more electron deficient KATs are more reactive overall, but that the presence of a proton near the KAT groups greatly accelerates the reaction and becomes the determining factor at higher $\mathrm{pH}$ regimes.

(a)

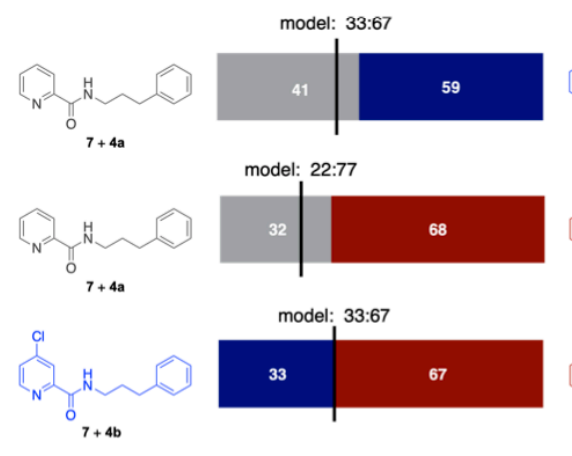

(b)
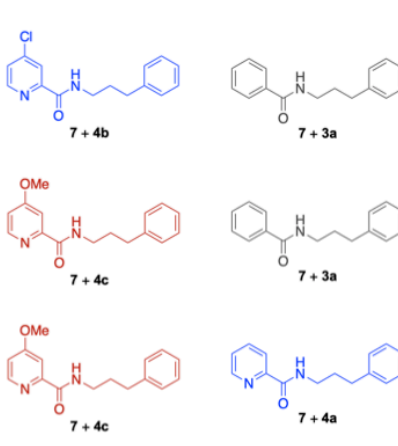
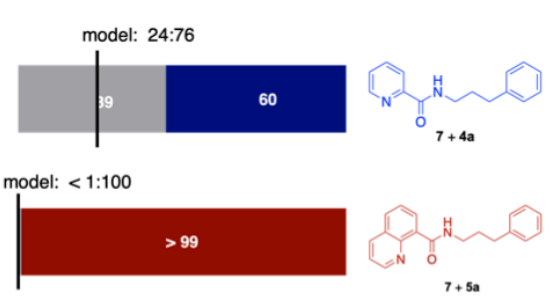
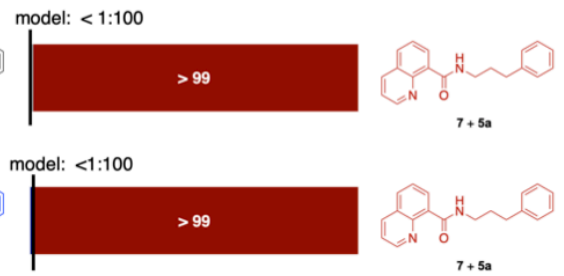

Figure 3: (a) Competitive ligation of KAT $\mathbf{4 a}, \mathbf{4 b}$, and $\mathbf{4 c}$ with hydroxylamine 7 at $\mathrm{pH}$ 7.4. Colored bars at top, middle, and bottom indicate the product distribution from $\mathbf{4 a}$ versus $\mathbf{4 b}$, $\mathbf{4 a}$ versus $\mathbf{4 c}$ and $\mathbf{4 b}$ versus $\mathbf{4 c}$, respectively, with the product from $\mathbf{4 a}, \mathbf{4 b}$, and $\mathbf{4 c}$ represented in grey, blue and red. The product distribution was measured by ${ }^{1} \mathrm{H}-\mathrm{NMR}$ and the vertical black mark indicated modeling results from the measured rate constants. (b) Competitive ligation of KAT 3a, $\mathbf{4 a}$, and $\mathbf{5 a}$.

This UV-vis method was able to determine the reaction rate constants of KATs with hydroxylamine 6 by monitoring the consumption of KATs, rather than the formation of the ligation product amide. To verify that the rate of product formation correlated with the reactivity of the KATs, and not just 
a difference in the formation of an initial adduct, a competition experiment was designed. KATs $\mathbf{4 a}, \mathbf{4 b}$, and $\mathbf{4 c}$ with relative reactivities of $3.34,8.33$ and 16.7 to the reference at $\mathrm{pH} 7.4$, were selected for pairwise competition with a limited amount of hydroxylamine 7 and the final product distribution was measured by ${ }^{1} \mathrm{H}-\mathrm{NMR}$. The parallel competition outcomes were compared to modeled results based on the initial concentration of reactants and rate constants. The agreement of observed and predicted results are depicted in Figure 3 (see SI for model and parameters).

Mechanism of KAT ligations. Our basic understanding of the mechanism of KAT ligation featured a nucleophilic addition of the hydroxylamine to the KAT carbonyl, forming a tetrahedral intermediate, followed by its breakdown (Scheme 3). At the outset of our studies, however, we had little insight into the specific bond-forming and bond-breaking processes or the role of protonation in accelerating the KAT ligation.

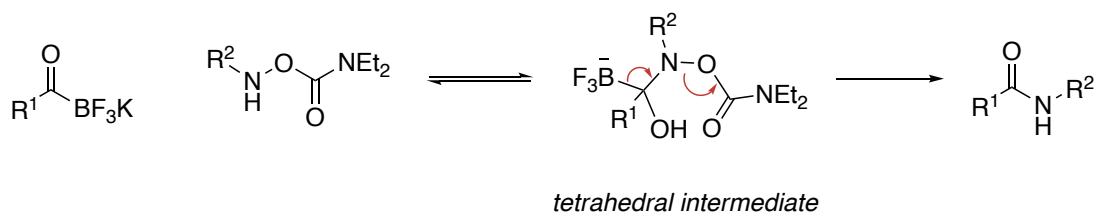

Scheme 3. Overview of KAT ligation reaction mechanism.

To gain further insights into possible mechanisms for the KAT ligation, we performed DFT calculations (def2/ma-TZVP) with $\mathrm{ORCA}^{24,25}$ on both protonated and unprotonated reaction pathways of KAT 4a, with the obtained structure and free energies depicted in Scheme 4. Comparison of the protonated reaction pathway (I-b - TS-b) and the unprotonated pathway (I-a - TS-a) suggested that the presence of a proton not only lowered the activation barrier from intermediates I-a and I-b to the corresponding transition states, but also took part in fixing the rotation along the hydroxylamine $\mathrm{N}-\mathrm{O}$ bond (in blue) in I-b. This resulted in a more rigid intermediate structure through hydrogen bond network around the hemiaminal hydroxyl group I-b. Nudged Elastic Band (NEB) computation indicated that throughout the reaction course from I-b to TS-b, protonation of the carbamoyl oxygen by the pyridinium proton occurred concomitantly with the cleavage of the hydroxylamine $\mathrm{N}-\mathrm{O}$ bond as well as that of the trifluoroborate $\mathrm{C}-\mathrm{B}$ bond, leading to a $\mathrm{C}$ to $\mathrm{N}$ 1,2- $\mathrm{BF}_{3}$ migration. ${ }^{26}$ 1,2-migration of the tetracoordinated boron group to carbon, nitrogen, and oxygen atoms has been shown for B-MIDA ( $N$-methyliminodiacetic acid) 
acylboronates. ${ }^{27}$ These migrations proceeded via a tetrahedral intermediate, where a leaving group at the $\beta$-position of the boronate initiated the migration (Scheme 5 ). The stereochemistry between the leaving group and the boronate group has also been found to effect the migratory aptitude of the boronate group. ${ }^{27}$ The computed reaction pathways shown in Scheme 4 led to the formation of an $\mathrm{N}_{-} \mathrm{BF}_{3}$ amide as the primary ligation product, which was also observed in real time mass spectrometry analysis of the reaction mixture (see Supporting Information Fig. 22).
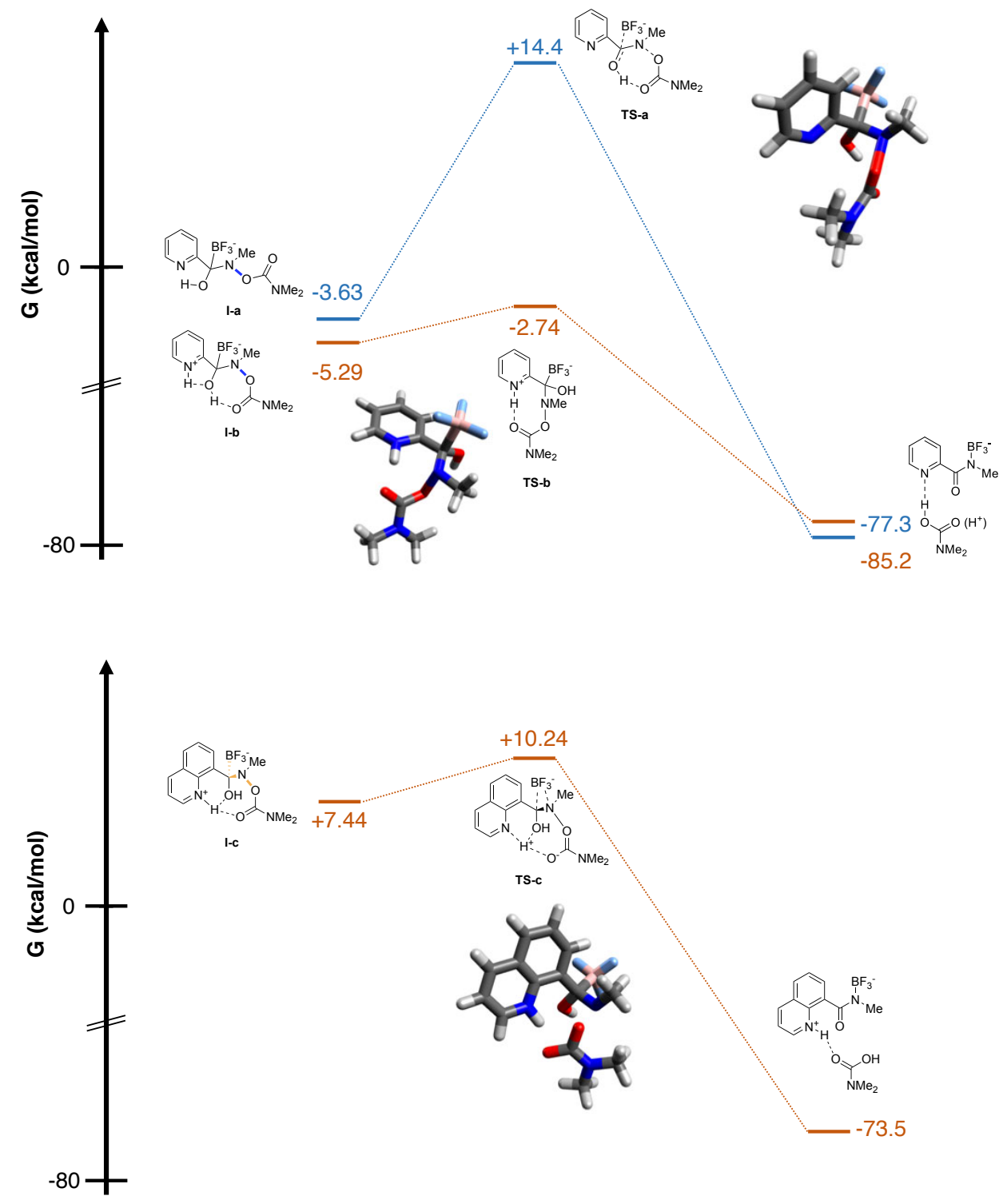
Scheme 4. Computed KAT ligation reaction of KATs $\mathbf{4 a}$ and $\mathbf{5 a}$. Reaction paths with the pyridine or quinoline being protonated were colored in brown. The vertical axes between 0 and $-80 \mathrm{kcal} / \mathrm{mol}$ were truncated to accommodate the free energy of the reaction products. Sums of free energies of the hydroxylamine and (protonated) KATs were set to zero as references. Computations were performed at the def2/ma-TZVP level. ${ }^{24,25}$
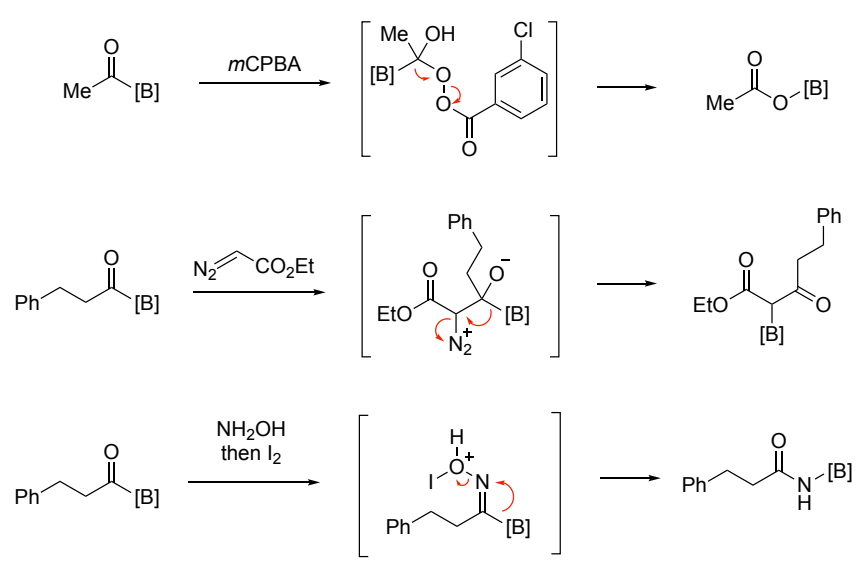

Scheme 5. Instances of 1,2-migration of boronate groups in B-MIDA acylboronates reported by Yudin. ${ }^{27}$ $[\mathrm{B}]=\mathrm{B}-\mathrm{N}-$ Methyliminodiacetate.

In the computed ligation pathway of quinolyl KAT 5a, quinoline protonation also favored the formation of a hydrogen bond-tethered intermediate I-c. The quinolinium proton was in vicinity to three basic atoms: the quinoline nitrogen, the hemiaminal oxygen, and the carbamoyl oxygen. Interaction between the proton and these atoms fixed the $\mathrm{F}_{3} \mathrm{~B}-\mathrm{C}-\mathrm{N}-\mathrm{O}$ dihedral angle (marked in orange), across the $\mathrm{C}-\mathrm{N}$ bond between the KAT carbonyl and hydroxylamine nitrogen, close to an antiperiplanar $180^{\circ}$. This conformation would facilitate the concerted $\mathrm{C}-\mathrm{B}$ bond / $\mathrm{N}-\mathrm{O}$ bond cleavage towards the transition state. The activation barrier from intermediate l-c, formed from protonated KAT 5a, to TS-c, was found to be slightly higher than that of from I-b to TS-b. This corresponded to the slightly higher observed rate of 4 a ligating at $\mathrm{pH} 3.8$, where both KATs are mostly protonated.

Nitrogen containing heteroaryl aldehydes are known to participate in reactions of neighboring groups. $^{28-30}$ For example, pyridine-2-carbaldehyde and quinoline-8-carbaldehyde form hydrazones faster than their corresponding hydrocarbon variants, benzaldehydes and naphthaldehydes. Kool et al. proposed that an intramolecular proton donor could facilitate both 
the formation and breakdown of the tetrahedral intermediate. ${ }^{28}$ Our findings suggest that a similar mode of rate acceleration was operative in KAT ligations, especially for quinolyl KATs $\mathbf{5}$ - with some qualifications.

Quinolyl KATs had faster ligation rates than pyridyl KATs at neutral $\mathrm{pH}$. This suggested counterintuitively - that quinolyl KATs were protonated to a greater extent than their pyridine counterparts, as pyridine $\left(\mathrm{pK}_{\mathrm{a}}=5.2\right)$ is known to be more basic than quinoline $\left(\mathrm{pK}_{\mathrm{a}}=4.9\right)$. To examine further, we performed titrations of the KATs with hydrochloric acid in aqueous acetonitrile to measure their basicities, and determined that the conjugate acid of quinolyl KAT $5 \mathbf{a}$ has a pKa of 5.96, while the conjugate acid of pyridyl KAT $4 a$ has a lower $\mathrm{pK}_{\mathrm{a}}$ of 4.92 .

The inversion of the inherent basicity of pyridyl- and quinolyl KATs implies that the proximal KAT group facilitates the protonation of quinoline KATs, possibly due to the formation of an internal salt resulting in a zwitterionic species that does not require the potassium gegenion. To investigate this further, we grew crystals of various quinolyl- and pyridyl KATs from either $\mathrm{pH} 7.4$ buffers or dilute $\mathrm{HCl}$ solutions and determined their structures by X-ray diffraction. In the case of quinolyl KAT 5a, crystals grown from either dilute $\mathrm{HCl}$ or from $\mathrm{pH} 7.4$ buffer both delivered the protonated, zwitterionic form (Figure 4a). Notably, electronic rich quinolyl KAT $5 \mathbf{d}$ also crystalized as the protonated form, while the chloro derivative $\mathbf{5 b}$ afforded unprotonated potassium salts from the same buffer. In contrast, all pyridine KAT crystals were obtained as their potassium salts. The carbonyl group for the zwitterionic species $\mathbf{5 a}$ is almost coplanar to the aromatic ring while KAT 5b shows the highest torsional angle between the carbonyl group and its aryl ring (Figure 4b). 
(a)

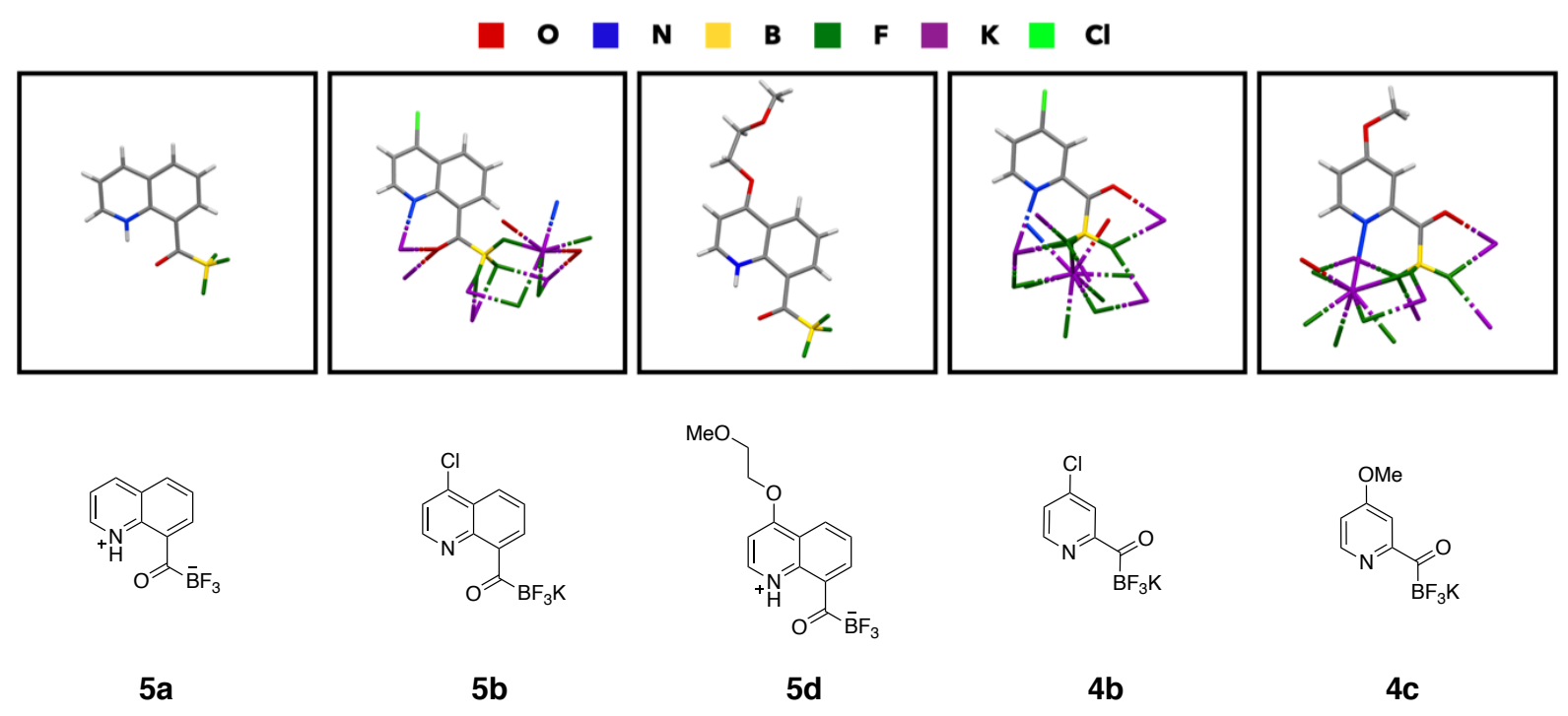

(b)

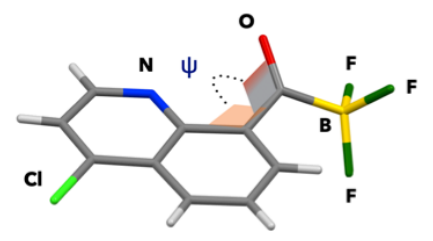

\begin{tabular}{c|c} 
Entry & $\Psi\left[^{\circ}\right]$ \\
\hline $\mathbf{5 a}$ & 6.48 \\
$\mathbf{5 b}$ & 35.15 \\
$\mathbf{5 d}$ & 18.81
\end{tabular}

(c)

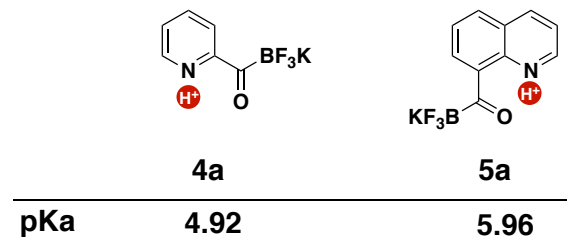

Figure 4. (a) Crystal structure of quinoline KATs $\mathbf{5 a}, \mathbf{5 b}, \mathbf{5} \mathbf{d}$ and pyridyl KATs $\mathbf{4 b}$ and $\mathbf{4 c}$. Quinolyl KATs were crystallized from potassium phosphate buffer $\mathrm{pH}$ 7.4. Atoms colored in yellow: boron, green: fluorine, purple: potassium, red: oxygen, light green: chlorine, blue: nitrogen. (b) Torsion angle between KAT carbonyl group and the plane of the quinoline backbone of $\mathbf{5 a}, \mathbf{5 b}$ and $\mathbf{5 d}$. (c) pK $\mathrm{K}_{\mathrm{a}}$ values of pyridyl KAT $\mathbf{4 a}$ and quinolyl KAT 5a. Titration curves are depicted in the SI.

The computational investigations indicated the importance of carbamoyl protonation during the $\mathrm{N}-\mathrm{O}$ bond cleavage during KAT ligation. This is consistent with the observation that all KATs ligate faster at lower $\mathrm{pH}$ and follow specific acid catalysis. ${ }^{31}$ The phenyl KATs 3a-c showed rate constants of $\sim 3 \mathrm{M}^{-1} \mathrm{~s}^{-1}$ at $\mathrm{pH} 3.8$. For KATs 4 and 5 , protonation is likely to both increase their electrophilicity and accelerate the $\mathrm{N}-\mathrm{O}$ bond cleavage by protonation of the carbamate, increasing the ligation rates to around $10 \mathrm{M}^{-1} \mathrm{~s}^{-1}$ at the same $\mathrm{pH}$. The more basic quinoline KATs 5a-d will have higher protonated fractions at neutral $\mathrm{pH}$ than pyridyl KATs 4 , and are more likely 
to form the intermediates leading to transition states. Consistent with this, KAT $\mathbf{5 d}$ with an alkoxy chain was by far the fastest ligating KAT at $\mathrm{pH} 7.4$.

KAT Ligations on Folded Proteins at pH 7.4. At the outset of this project, we sought to identify KAT ligation partners that formed amide bonds under physiological conditions with rate constants of at least $1 \mathrm{M}^{-1} \mathrm{~s}^{-1}$, which would make it suitable for bioconjugations at micromolar concentrations and potential in vivo applications. Although detailed applications of the quinolyl KATs to these goals are beyond the scope of this manuscript, we have confirmed the benefit of higher ligation rates of quinolyl KATs for protein modification.

According to our previously reported protocol, a sfGFP(S147C) mutant was expressed from chemically competent Escherichia coli BL21(DE3) cells in high yields and treated with the thiophilic hydroxylamine derivative. ${ }^{4}$ A $100 \mu \mathrm{M}$ solution of the sfGFP(S147C)-hydroxylamine adduct 8 was treated with quinolyl KAT $5 \mathbf{d}$ (5.0 equiv) at pH 7.4 in the same buffer system used for the kinetic measurements and was fully converted to the amide product 9 within 90 min. In contrast, the same experiment with pyridyl KAT 4a returned only unreacted protein after 90 min at $\mathrm{pH} 7.4$ (Figure 5). The reaction mixture was passed through a size-exclusion column to elute the protein in MilliQ water prior to mass analysis (ESI-MS). 
(a)

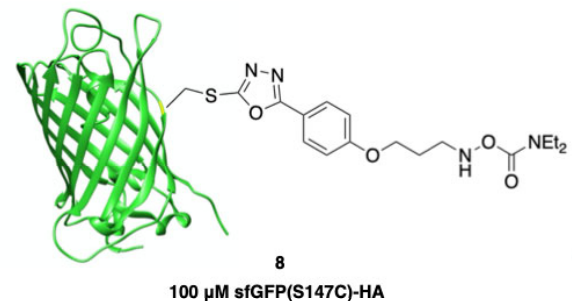

$100 \mu \mathrm{M}$ sfGFP(S147C)-HA

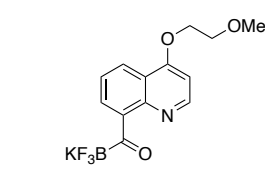

5d (5.0 equiv)

pH 7.4, $90 \mathrm{~min}$ 0.1 M KPhos buffer:MeCN 1:1 (v/v)

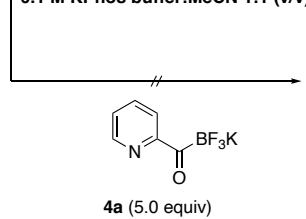

4a (5.0 equiv)

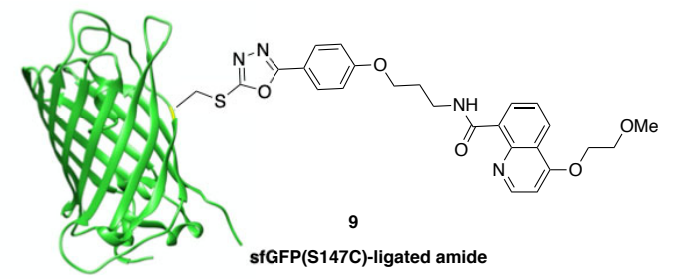

no product formed with 4 a

(b)
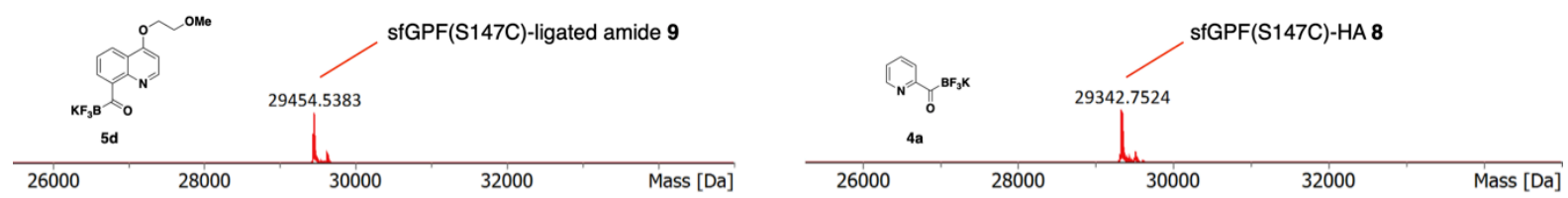

Figure 5. (a) Comparison between pyridyl KAT 4a and quinolyl KAT 5d with a model protein at $100 \mu \mathrm{M}$ protein concentration at $\mathrm{pH}$ 7.4. 5.0 equiv of KAT was added. The reaction mixture was stirred for 90 min at rt. (b) Deconvoluted mass spectra (ESI-MS) of each reaction mixture after size-exclusion column purification.

\section{Conclusion}

In summary, we have identified 8-quinolyl KATs as uniquely suited for rapid amide-forming ligations at $\mathrm{pH}$ 7.4. They exhibit second order rate constants of $1-5 \mathrm{M}^{-1} \mathrm{~s}^{-1}$ at physiological $\mathrm{pH}$, hundreds of times faster than previously examined KATs. The origin of the enhanced reactivity was traced - through kinetic studies, titrations, X-ray crystallography, and DFT calculations - to an unexpectedly high basicity of the quinolyl KATs and a key role for the proton in the mechanism of the KAT ligation. These findings were translated to ligation of a model protein at micromolar concentration in $\mathrm{pH} 7.4$ buffer and pave the way for applications of KAT ligation on systems that require rapid ligations at physiological $\mathrm{pH}$. 


\title{
Associated Content:
}

Accession Codes:

CCDC Deposition Numbers for X-ray structures are listed in the Supporting Information.

\section{Author Information}

\section{Corresponding Author}

Jeffrey W. Bode - Department of Chemistry and Applied Biosciences, ETH Zürich, Zürich 8093, Switzerland

\author{
Authors \\ Matthias Tanriver - Department of Chemistry and Applied Biosciences, ETH Zürich, Zürich 8093, \\ Switzerland
}

Yi-Chung Dzeng - Department of Chemistry and Applied Biosciences, ETH Zürich, Zürich 8093, Switzerland

Erwin Lam - Department of Chemistry and Applied Biosciences, ETH Zürich, Zürich 8093, Switzerland

\section{Author Contributions}

M.T. and Y.D. contributed equally.

\section{$\underline{\text { Notes }}$}

The authors declare no competing financial interest.

\section{Acknowledgements}

This work was financially supported by ETH Grants ETH-43 13-2 and ETH-44 17-2. Sara Da Ros is acknowledged for initial studies. We thank Gaku Akimoto, Kateryna Tolmachova and Dr. Chongqing Wang for their advice regarding protein purifications and for fruitful discussions. We acknowledge Prof. Simon Ametamey and Dr. Hazem Ahmed for helpful discussions, and we are grateful to Prof. Ken Houk for preliminary discussions regarding DFT calculations. 


\section{References}

1. Dumas, A. M.; Molander, G. A.; Bode, J. W., Amide-Forming Ligation of Acyltrifluoroborates and Hydroxylamines in Water. Angewandte Chemie International Edition 2012, 51 (23), 5683-5686.

2. Noda, H.; Erős, G.; Bode, J. W., Rapid Ligations with Equimolar Reactants in Water with the Potassium Acyltrifluoroborate (KAT) Amide Formation. Journal of the American Chemical Society 2014, 136 (15), 5611-5614.

3. Molander, G. A.; Raushel, J.; Ellis, N. M., Synthesis of an Acyltrifluoroborate and Its Fusion with Azides To Form Amides. The Journal of Organic Chemistry 2010, 75 (12), 4304-4306.

4. White, C. J.; Bode, J. W., PEGylation and Dimerization of Expressed Proteins under Near Equimolar Conditions with Potassium 2-Pyridyl Acyltrifluoroborates. ACS Central Science 2018, 4 (2), 197-206.

5. Mazunin, D.; Broguiere, N.; Zenobi-Wong, M.; Bode, J. W., Synthesis of Biocompatible PEG Hydrogels by $\mathrm{pH}$-Sensitive Potassium Acyltrifluoroborate (KAT) Amide Ligations. ACS Biomaterials Science \& Engineering 2015, 1 (6), 456-462.

6. Mazunin, D.; Bode, J. W., Potassium Acyltrifluoroborate (KAT) Ligations are Orthogonal to Thiol-Michael and SPAAC Reactions: Covalent Dual Immobilization of Proteins onto Synthetic PEG Hydrogels. Helvetica Chimica Acta 2017, 100 (2), e1600311.

7. Schauenburg, D.; Divandari, M.; Neumann, K.; Spiegel, C. A.; Hackett, T.; Dzeng, Y.C.; Spencer, N. D.; Bode, J. W., Synthesis of Polymers Containing Potassium Acyltrifluoroborates (KATs) and Post-polymerization Ligation and Conjugation. Angewandte Chemie International Edition 2020, 59 (34), 14656-14663.

8. Saito, F.; Noda, H.; Bode, J. W., Critical Evaluation and Rate Constants of Chemoselective Ligation Reactions for Stoichiometric Conjugations in Water. ACS Chemical Biology 2015, 10 (4), 1026-1033.

9. Wang, H.; Wang, R.; Cai, K.; He, H.; Liu, Y.; Yen, J.; Wang, Z.; Xu, M.; Sun, Y.; Zhou, X.; Yin, Q.; Tang, L.; Dobrucki, I. T.; Dobrucki, L. W.; Chaney, E. J.; Boppart, S. A.; Fan, T. M.; Lezmi, S.; Chen, X.; Yin, L.; Cheng, J., Selective in vivo metabolic celllabeling-mediated cancer targeting. Nature Chemical Biology 2017, 13 (4), 415-424.

10. Chang, P. V.; Prescher, J. A.; Sletten, E. M.; Baskin, J. M.; Miller, I. A.; Agard, N. J.; Lo, A.; Bertozzi, C. R., Copper-free click chemistry in living animals. Proceedings of the National Academy of Sciences 2010, 107 (5), 1821-1826. 
11. Erős, G.; Kushida, Y.; Bode, J. W., A Reagent for the One-Step Preparation of Potassium Acyltrifluoroborates (KATs) from Aryl- and Heteroarylhalides. Angewandte Chemie International Edition 2014, 53 (29), 7604-7607.

12. Cheng, L.-J.; Zhao, S.; Neal, M., One-Step Synthesis of Acylboron Compounds via CuCatalyzed Carbonylative Borylation of Alkyl Halides. 2020.

13. Liu, S. M.; Mazunin, D.; Pattabiraman, V. R.; Bode, J. W., Synthesis of Bifunctional Potassium Acyltrifluoroborates. Organic Letters 2016, 18 (20), 5336-5339.

14. Liu, S. M.; Wu, D.; Bode, J. W., One-Step Synthesis of Aliphatic Potassium Acyltrifluoroborates (KATs) from Organocuprates. Organic Letters 2018, 20 (8), 23782381.

15. Taguchi, J.; Takeuchi, T.; Takahashi, R.; Masero, F.; Ito, H., Concise Synthesis of Potassium Acyltrifluoroborates from Aldehydes through Copper(I)-Catalyzed Borylation/Oxidation. Angewandte Chemie International Edition 2019, 58 (22), 7299-7303.

16. Wu, D.; Fohn, N. A.; Bode, J. W., Catalytic Synthesis of Potassium Acyltrifluoroborates (KATs) through Chemoselective Cross-Coupling with a Bifunctional Reagent. Angewandte Chemie International Edition 2019, 58 (32), 11058-11062.

17. Dumas, A. M.; Bode, J. W., Synthesis of Acyltrifluoroborates. Organic Letters 2012, 14 (8), 2138-2141.

18. Schuhmacher, A.; Ryan, S. J.; Bode, J. W., Catalytic Synthesis of Potassium Acyltrifluoroborates (KATs) from Boronic Acids and the Thioimidate KAT Transfer Reagent. Angewandte Chemie International Edition 2020.

19. Cheng, L.-J.; Zhao, S.; Mankad, N. P., One-Step Synthesis of Acylboron Compounds via Copper-Catalyzed Carbonylative Borylation of Alkyl Halides**. Angewandte Chemie International Edition 2021, 60 (4), 2094-2098.

20. Sawada, M.; Ichihara, M.; Yukawa, Y.; Nakachi, T.; Tsuno, Y., The Substituent Effect. 15. The LSFE Treatment of Substituent Effects on Basicities of Pyridines. In Bulletin of the Chemical Society of Japan, 1980; Vol. 53, pp 2055-2060.

21. Taagepera, M.; Henderson, W. G.; Brownlee, R. T. C.; Beauchamp, J. L.; Holtz, D.; Taft, R. W., Gas-Phase Basicities and Pyridine Substituent Effects. In Journal of the American Chemical Society, 1972; Vol. 94, pp 1369-1370.

22. Albert, A.; Goldacre, R.; Phillips, J., 455. The strength of heterocyclic bases. Journal of the Chemical Society (Resumed) 1948, (0), 2240-2249.

23. Gero, A.; Markham, J. J., Studies on Pyridines: I. The Basicity of Pyridine Bases. The Journal of Organic Chemistry 1951, 16 (12), 1835-1838.

24. Neese, F., The ORCA program system. WIREs Computational Molecular Science 2012, 2 (1), 73-78. 
25. Neese, F., Software update: the ORCA program system, version 4.0. WIREs Computational Molecular Science 2018, 8 (1), e1327.

26. He, Z.; Yudin, A. K., Amphoteric a-Boryl Aldehydes. Journal of the American Chemical Society 2011, 133 (35), 13770-13773.

27. Lee, C. F.; Diaz, D. B.; Holownia, A.; Kaldas, S. J.; Liew, S. K.; Garrett, G. E.; Dudding, T.; Yudin, A. K., Amine hemilability enables boron to mechanistically resemble either hydride or proton. Nature Chemistry 2018, 10 (10), 1062-1070.

28. Kool, E. T.; Park, D.-H.; Crisalli, P., Fast Hydrazone Reactants: Electronic and Acid/Base Effects Strongly Influence Rate at Biological pH. Journal of the American Chemical Society 2013, 135 (47), 17663-17666.

29. Lord, A.-M.; Mahon, M. F.; Lloyd, M. D.; Threadgill, M. D., Design, Synthesis, and Evaluation in Vitro of Quinoline-8-carboxamides, a New Class of Poly(adenosinediphosphate-ribose)polymerase-1 (PARP-1) Inhibitor. Journal of Medicinal Chemistry 2009, 52 (3), 868-877.

30. Higgs, P. L.; Ruiz-Sanchez, A. J.; Dalmina, M.; Horrocks, B. R.; Leach, A. G.; Fulton, D. A., Enhancing the kinetics of hydrazone exchange processes: an experimental and computational study. Organic \& Biomolecular Chemistry 2019, 17 (12), 3218-3224.

31. Da Ros, S. Kinetic and Mechanistic Investigation of the Potassium Acyltrifluoroborate Ligation Reaction. ETH Zurich, 2018. 